OPEN ACCESS

Edited by:

Aali Jan Sheen,

Manchester Royal Infirmary,

United Kingdom

Reviewed by:

Enrico Checcucci,

IRCCS Candiolo Cancer Institute, Italy

Patrick Richard,

Centre Hospitalier Universitaire de

Sherbrooke, Canada

*Correspondence:

Chunguang Yang

cgyang-hust@hotmail.com

Zhiquan Hu

huzhiquan2000@163.com

Specialty section:

This article was submitted to

Surgical Oncology,

a section of the journal

Frontiers in Oncology

Received: 20 November 2021

Accepted: 30 December 2021

Published: 26 January 2022

Citation:

Tian J, Zeng X, Wan J, Gan J, Ke C,

Guan W, Hu Z and Yang C (2022)

Partial and Radical Nephrectomy

Provides Equivalent Oncologic

Outcomes in pT3a Renal

Cell Carcinoma:

A Population-Based Study.

Front. Oncol. 11:819098.

doi: 10.3389/fonc.2021.819098

\section{Partial and Radical Nephrectomy Provides Equivalent Oncologic Outcomes in pT3a Renal Cell Carcinoma: A Population-Based Study}

\author{
Jihua Tian ${ }^{1}$, Xing Zeng ${ }^{1}$, Jie Wan ${ }^{2}$, Jiahua Gan ${ }^{1}$, Chunjin $\mathrm{Ke}^{1}$, Wei Guan ${ }^{1}$, \\ Zhiquan $\mathrm{Hu}^{1 *}$ and Chunguang Yang ${ }^{1 *}$ \\ ${ }^{1}$ Department of Urology, Tongji Hospital of Tongji Medical College, Huazhong University of Science and Technology (HUST), \\ Wuhan, China, ${ }^{2}$ Department of Pathology, Tongji Hospital of Tongji Medical College, Huazhong University of Science and \\ Technology, Wuhan, China
}

Purpose: To compare the cause-specific survival (CSS) and overall survival (OS) of patients with localized T3a renal cell carcinoma (RCC) after partial nephrectomy (PN) or radical nephrectomy $(\mathrm{RN})$.

Methods: We obtained the demographic and clinicopathological data of 7,127 patients with localized $\mathrm{T} 3 \mathrm{a} \mathrm{RCC}$ and who underwent PN or RN from the Surveillance, Epidemiology, and End Results (SEER) database. These patients were divided into fat invasion cohort and venous invasion cohort for subsequent analysis. Kaplan-Meier analysis (KMA) and univariate and multivariate Cox proportional hazards regression analyses were used to evaluate the effects of PN or RN on OS and CSS. Meanwhile, 65 cases with clinical T1 (cT1) RCC upstaged to pathological T3a (pT3a) who were treated in Tongji Hospital (TJH) from 2011 to 2020 and underwent PN or RN were identified.

Results: In the study cohort, 2,085 (29.3\%) patients died during the 1-172 months' follow-up, of whom 1,155 (16.2\%) died of RCC. In the two cohorts of fat invasion and venous invasion, KMA indicated that the PN group had favorable survival $(p<0.001)$. However, after propensity score matching (PSM), univariate and multivariate Cox regression analyses showed that the $\mathrm{PN}$ and RN groups had comparable CSS in the fat invasion cohort $(p=0.075)$ and the venous invasion cohort $(p=0.190)$. During $1-104$ months of follow-up, 9 cases in the Tongji cohort had disease recurrence. There was no significant difference in recurrence-free survival between the $\mathrm{RN}$ group and the PN group $(p=0.170)$.

Conclusions: Our analysis showed that after balancing these factors, patients with localized pT3a RCC receiving PN or RN can achieve comparable oncologic outcomes. PN is safe for selected T3a patients.

Keywords: partial nephrectomy, radical nephrectomy, renal cell carcinoma, outcomes, T3a 


\section{INTRODUCTION}

Renal cell carcinoma (RCC) accounts for 2\%-3\% of all adult malignancies (1). In recent years, with the wide applications of imaging examinations, the proportion of early-staged RCC has gradually increased (2), and partial nephrectomy (PN) has therefore played a more important role in the treatment of $\operatorname{RCC}(3,4)$.

$\mathrm{PN}$ is currently the standard treatment for T1 RCC, which provides similar oncologic control to radical nephrectomy (RN) while reducing the loss of renal function $(5,6)$. The current guidelines for $\mathrm{PN}$ recommendations are limited to $\mathrm{T} 1$ and selected T2 RCC (combined with solitary kidney or chronic kidney disease if technically feasible) $(5,7,8)$. However, in clinical practice, patients with clinical T1 (cT1) upstaged to pathological T3a (pT3a) RCC underwent PN did not show unfavorable cause-specific survival (CSS) and recurrence-free survival (RFS) than did those who received RN, which led us to think about the safety of PN for selected T3a patients $(9,10)$.

In the past 10 years, the application of PN in T3a RCC patients has been explored, more and more evidences show that $\mathrm{PN}$ is safe and feasible for some T3a cases (10-13), and there are also dissenting voices suggesting that $\mathrm{PN}$ is associated with poor oncologic outcome (14). Most of these studies are small-volume and retrospective. To this end, we selected the Surveillance, Epidemiology, and End Results (SEER) database to compare the performance of PN and RN in T3a RCC patients and used propensity score matching (PSM) to control bias; finally, we attached data of cT1 RCC patients upstaged to pT3a from our institution to add new evidence to this controversy.

\section{PATIENTS AND METHODS}

SEER is a population-based cancer database found by the National Cancer Institute that collects data on morbidity, treatment, and mortality (15). We obtained data of RCC patients diagnosed from 2004 to 2015 from 18 registries of the SEER database. The process of screening patients was shown below (Figure 1).

The inclusion criteria of the study were as follows: malignant pathological diagnosis; age 15 years and above; histologic type is renal cancer (including clear cell RCC (CCRCC), RCC not otherwise specified (NOS), papillary RCC (PRCC), acquired cystic disease-associated RCC/tubulocystic RCC, chromophobe RCC (CHRCC), clear cell PRCC, collecting duct carcinoma, hereditary leiomyomatosis and RCC-associated RCC, MiT family translocation RCC, mucinous tubular and spindle cell carcinoma, and renal medullary carcinoma); staged T3a; no lymph nodes and distant metastasis; no bilateral or other tumors; the surgical method was PN or RN; follow-up longer than 1 month; and knowable survival status.

The histologic types were divided into CCRCC, PRCC, CHRCC, and Others. According to the tissues of tumor invasion, the study cohort was divided into the fat invasion and venous invasion cohorts. The demographic and clinicopathological data of the RN and PN groups in the two cohorts were analyzed separately. The continuous variables were analyzed by Student's t-test, and the categorical variables were analyzed by Fisher's exact test. Then, a 1:1 PSM was performed between the surgical methods (PN vs. RN); all demographic and clinicopathological features were used as calipers (half of the PN cases in the fat invasion cohort were randomly rejected because of lack of enough $\mathrm{RN}$ cases to match).

The Kaplan-Meier analysis (KMA) was used to evaluate the survival difference between the $\mathrm{RN}$ and $\mathrm{PN}$ groups in the venous invasion cohort and the fat invasion cohort, respectively. Then univariate and multivariate Cox regression analyses were used to analyze the effect of each factor on survival. The variables of multivariate Cox regression were derived from the significant prognostic factors of univariate Cox regression.

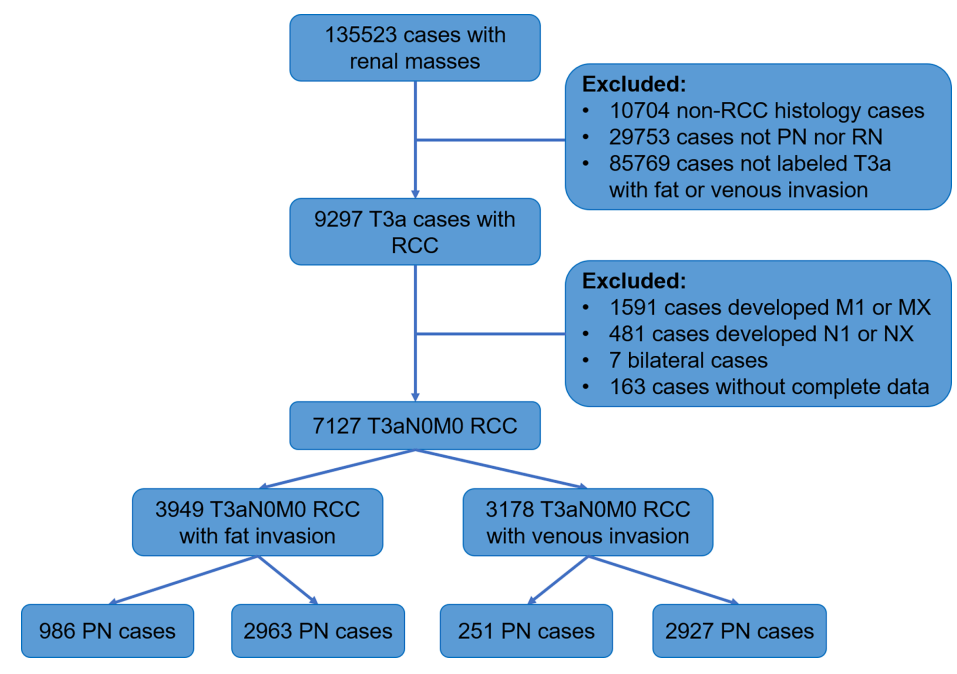

FIGURE 1 | Flowchart displaying patient selection procedure. 
Finally, after institutional review board approval, a retrospective review of all patients with upstaged pT3a RCC managed with PN or RN (2010.1.1-2020.12.1) in the Department of Urology of Tongji Hospital of Tongji Medical College, Huazhong University of Science and Technology (HUST) was performed $(n=65)$. KMA and univariate and multivariate Cox regression analyses were also performed in this cohort. All the above analyses were performed by $\mathrm{R}$ version 4.0.3 (Institute for Statistics and Mathematics, Vienna, Austria; www.r-project.org), and $p<0.05$ was considered statistically significant.

\section{RESULTS}

After the screening procedure, 7,127 T3aN0M0 RCC cases (Table 1) were obtained. The median age was 62 years (22-87 years). There were 1,237 cases $(17.4 \%)$ who received PN. The maximum diameter of tumors in the PN group was significantly smaller than that of the $\mathrm{RN}$ group (40 [29.00, 53.00] mm vs. 74 $[55.00,95.00] \mathrm{mm}, p<0.001)$. There were also significant differences in histologic types and nuclear grade structure between the two groups (both $p<0.001$ ). The proportion of CCRCC and high-grade RCC in the RN group was higher. The median follow-up time was 56 months (1-172 months); 2,085 cases $(29.3 \%)$ were confirmed dead at the last follow-up, of whom 1,205 cases (60.4\%) died of RCC; the 1-, 3-, and 5-year survival rates were $95.1 \%, 83.4 \%$, and $73.8 \%$, respectively.

The study cohort was divided into 3,949 cases of fat invasion (including 986 cases of $\mathrm{PN}$ and 2,963 cases of $\mathrm{RN}$ ) and 3,178 cases of venous invasion (including 251 cases of PN and 2,927 cases of RN). The demographics and clinicopathological data of the RN group and the PN group in the two cohorts showed significant differences (Tables 2, 3). KMA showed that the overall survival (OS) and CSS of the PN groups were favorable than those of the RN groups in the two cohorts (both $p<0.001$ ) (Figure 2). After PSM, there was no significant difference in the characteristics between the PN and RN groups in the two cohorts (Tables 2, 3). There was no significant difference in OS ( $p=$ $0.068)$ and CSS $(p=0.190)$ between the PN and RN groups in the venous invasion cohort. The OS in the fat invasion group was favorable in the PN group ( $p=0.036)$, while CSS was comparable in the two groups $(p=0.075)$ (Figure 3).

Univariate and multivariate regression analyses showed that the risk factors of OS in the fat invasion cohort were higher age (hazard ratio (HR) 1.05, $p<0.001$ ), greater tumor diameter (HR $1.01, p=0.007)$, surgical method was $\mathrm{RN}(\mathrm{HR} 1.35, p=0.040$ ), and earlier year of diagnosis (HR 1.10, $p=0.032$ ); nuclear grade is also an independent prognostic factor $(p=0.016)$ (Table 4). The risk factors for CSS in the fat invasion cohort were higher

TABLE 1 | Characteristics of PN and RN groups in SEER cohorts.

\begin{tabular}{|c|c|c|c|}
\hline Characteristics & PN $(n=1,237)$ & RN (n = 5,890) & $p$ \\
\hline Year of diagnosis (median [IQR]) & $2,013.00[2,011.00,2,014.00]$ & $2,013.00[2,011.00,2,014.00]$ & $<0.001$ \\
\hline Age (median [IQR]) & $62.00[57.00,72.00]$ & $62.00[57.00,72.00]$ & 0.013 \\
\hline Size (median [IQR]) & $40.00[29.00,53.00]$ & $74.00[55.00,95.00]$ & $<0.001$ \\
\hline Grade (\%) & & & $<0.001$ \\
\hline 1 & $68(5.5)$ & $166(2.8)$ & \\
\hline$\|$ & $508(41.1)$ & $1,946(33.0)$ & \\
\hline III & $414(33.5)$ & 2,303 (39.1) & \\
\hline IV & $73(5.9)$ & $763(13.0)$ & \\
\hline Unknown & $174(14.1)$ & $712(12.1)$ & \\
\hline Race (\%) & & & 0.012 \\
\hline Black & $111(9.0)$ & $397(6.7)$ & \\
\hline Other & $88(7.1)$ & $377(6.4)$ & \\
\hline White & 1,038 (83.9) & $5,116(86.9)$ & \\
\hline $\operatorname{Sex}(\%)$ & & & 0.162 \\
\hline Female & $352(28.5)$ & $1,796(30.5)$ & \\
\hline Male & $885(71.5)$ & 4,094 (69.5) & \\
\hline Histology (\%) & & & $<0.001$ \\
\hline CCRCC & 766 (61.9) & $4,544(77.1)$ & \\
\hline $\mathrm{CHRCC}$ & $119(9.6)$ & $312(5.3)$ & \\
\hline PRCC & 238 (19.2) & $323(5.5)$ & \\
\hline Others & $114(9.2)$ & $721(12.2)$ & \\
\hline Laterality (\%) & & & 0.080 \\
\hline Left & 598 (48.3) & $3,010(51.1)$ & \\
\hline Right & $639(51.7)$ & 2,880 (48.9) & \\
\hline Chemotherapy (\%) & & & $<0.001$ \\
\hline No/unknown & 1,215 (98.2) & 5,609 (95.2) & \\
\hline Yes & $22(1.8)$ & $281(4.8)$ & \\
\hline Extension (\%) & & & $<0.001$ \\
\hline Fat & 986 (79.7) & 2,963 (50.3) & \\
\hline VTT & 251 (20.3) & $2,927(49.7)$ & \\
\hline
\end{tabular}

PN, partial nephrectomy; RN, radical nephrectomy; SEER, Surveillance, Epidemiology, and End Results; IQR, interquartile range; CCRCC, clear cell renal cell carcinoma; CHRCC, chromophobe renal cell carcinoma; PRCC, papillary renal cell carcinoma; VTT, venous tumor thrombus. 
TABLE 2 | Characteristics of fat invasion cohort before and after PSM.

\begin{tabular}{|c|c|c|c|c|c|c|}
\hline \multirow[t]{2}{*}{ Characteristics } & \multicolumn{3}{|c|}{ Before match } & \multicolumn{3}{|c|}{ After match } \\
\hline & PN (n = 986) & $R N(n=2,963)$ & $p$ & PN $(n=496)$ & RN $(n=496)$ & $p$ \\
\hline Year of diagnosis (median [IQR]) & $2,013.00[2,012.00,2,014.00]$ & $2,013.00[2,011.00,2,014.00]$ & $<0.001$ & $2,013.00[2,012.00,2,014.00]$ & $2,013.00[2,011.00,2,014.00]$ & 0.977 \\
\hline Age (median [IQR]) & $62.00[57.00,72.00]$ & $62.00[57.00,72.00]$ & 0.140 & $62.00[57.00,72.00]$ & $67.00[57.00,72.00]$ & 0.274 \\
\hline Size (median [IQR]) & $38.00[27.00,50.00]$ & $70.00[50.00,92.00]$ & $<0.001$ & $38.00[26.00,50.00]$ & $40.00[28.00,50.00]$ & 0.503 \\
\hline Grade (\%) & & & $<0.001$ & & & 0.892 \\
\hline 1 & $59(6.0)$ & $105(3.5)$ & & $26(5.2)$ & $29(5.8)$ & \\
\hline$\|$ & $419(42.5)$ & $1,010(34.1)$ & & $212(42.7)$ & $200(40.3)$ & \\
\hline III & $306(31.0)$ & $1,097(37.0)$ & & $144(29.0)$ & $156(31.5)$ & \\
\hline IV & $61(6.2)$ & $343(11.6)$ & & $38(7.7)$ & $35(7.1)$ & \\
\hline Unknown & $141(14.3)$ & $408(13.8)$ & & $76(15.3)$ & $76(15.3)$ & \\
\hline Race (\%) & & & 0.042 & & & 0.437 \\
\hline Black & $93(9.4)$ & $220(7.4)$ & & $50(10.1)$ & $50(10.1)$ & \\
\hline Other & $75(7.6)$ & 189 (6.4) & & $38(7.7)$ & $28(5.6)$ & \\
\hline White & 818 (83.0) & $2,554(86.2)$ & & 408 (82.3) & 418 (84.3) & \\
\hline Sex (\%) & & & 0.275 & & & 0.186 \\
\hline Female & $274(27.8)$ & 879 (29.7) & & $136(27.4)$ & $156(31.5)$ & \\
\hline Male & 712 (72.2) & $2,084(70.3)$ & & 360 (72.6) & $340(68.5)$ & \\
\hline Histology (\%) & & & $<0.001$ & & & 0.975 \\
\hline CCRCC & $563(57.1)$ & 2,104 (71.0) & & $282(56.9)$ & $288(58.1)$ & \\
\hline CHRCC & $108(11.0)$ & $241(8.1)$ & & $57(11.5)$ & $53(10.7)$ & \\
\hline PRCC & 227 (23.0) & $253(8.5)$ & & 107 (21.6) & 105 (21.2) & \\
\hline Others & $88(8.9)$ & 365 (12.3) & & $50(10.1)$ & $50(10.1)$ & \\
\hline Laterality (\%) & & & 0.040 & & & 0.949 \\
\hline Left & $470(47.7)$ & $1,525(51.5)$ & & $245(49.4)$ & 247 (49.8) & \\
\hline Right & $516(52.3)$ & $1,438(48.5)$ & & 251 (50.6) & $249(50.2)$ & \\
\hline Chemotherapy (\%) & & & $<0.001$ & & & 0.328 \\
\hline No/unknown & $974(98.8)$ & 2,850 (96.2) & & 490 (98.8) & $485(97.8)$ & \\
\hline Yes & $12(1.2)$ & $113(3.8)$ & & $6(1.2)$ & $11(2.2)$ & \\
\hline
\end{tabular}

PSM, propensity score matching; PN, partial nephrectomy; RN, radical nephrectomy; IQR, interquartile range; CCRCC, clear cell renal cell carcinoma; CHRCC, chromophobe renal cell carcinoma; PRCC, papillary renal cell carcinoma.

TABLE 3 | Characteristics of venous invasion cohort before and after PSM.

\begin{tabular}{|c|c|c|c|c|c|c|}
\hline \multirow[t]{2}{*}{ Characteristics } & \multicolumn{3}{|c|}{ Before match } & \multicolumn{3}{|c|}{ After match } \\
\hline & PN (n = 251) & $\mathrm{RN}(\mathrm{n}=\mathbf{2 , 9 2 7 )}$ & $p$ & PN (n = 251) & RN (n = 251) & $p$ \\
\hline Year of Diagnosis (median [IQR]) & $2,013.00[2,011.00,2,014.00]$ & $2,013.00[2,011.00,2,014.00]$ & 0.566 & $2,013.00[2,011.00,2,014.00]$ & $2,013.00[2,011.00,2,014.00]$ & 0.937 \\
\hline Age (median [IQR]) & $62.00[57.00,72.00]$ & $62.00[57.00,72.00]$ & 0.058 & $62.00[57.00,72.00]$ & $62.00[57.00,72.00]$ & 0.515 \\
\hline Size (median [IQR]) & $46.00[35.00,67.50]$ & $76.00[57.00,100.00]$ & $<0.001$ & $46.00[35.00,67.50]$ & $50.00[35.00,70.00]$ & 0.741 \\
\hline Grade (\%) & & & $<0.001$ & & & 0.890 \\
\hline I & 9 (3.6) & $61(2.1)$ & & 9 (3.6) & $12(4.8)$ & \\
\hline$\|$ & 89 (35.5) & 936 (32.0) & & 89 (35.5) & $83(33.1)$ & \\
\hline III & $108(43.0)$ & $1,206(41.2)$ & & $108(43.0)$ & $111(44.2)$ & \\
\hline IV & $12(4.8)$ & $420(14.3)$ & & $12(4.8)$ & $15(6.0)$ & \\
\hline Unknown & $33(13.1)$ & $304(10.4)$ & & $33(13.1)$ & $30(12.0)$ & \\
\hline Race (\%) & & & 0.602 & & & 1.000 \\
\hline Black & $18(7.2)$ & $177(6.0)$ & & $18(7.2)$ & $18(7.2)$ & \\
\hline Other & $13(5.2)$ & $188(6.4)$ & & $13(5.2)$ & $14(5.6)$ & \\
\hline White & $220(87.6)$ & $2,562(87.5)$ & & $220(87.6)$ & $219(87.3)$ & \\
\hline Sex $(\%)$ & & & 1.000 & & & 0.774 \\
\hline Female & $78(31.1)$ & 917 (31.3) & & 78 (31.1) & $82(32.7)$ & \\
\hline Male & $173(68.9)$ & $2,010(68.7)$ & & $173(68.9)$ & $169(67.3)$ & \\
\hline Histology (\%) & & & 0.057 & & & 0.839 \\
\hline CCRCC & 203 (80.9) & $2,440(83.4)$ & & $203(80.9)$ & $200(79.7)$ & \\
\hline $\mathrm{CHRCC}$ & $11(4.4)$ & $71(2.4)$ & & $11(4.4)$ & $9(3.6)$ & \\
\hline PRCC & $11(4.4)$ & $70(2.4)$ & & $11(4.4)$ & $10(4.0)$ & \\
\hline Others & $26(10.4)$ & $346(11.8)$ & & $26(10.4)$ & $32(12.7)$ & \\
\hline Laterality (\%) & & & 0.948 & & & 0.858 \\
\hline Left & $128(51.0)$ & $1,485(50.7)$ & & $128(51.0)$ & $131(52.2)$ & \\
\hline Right & $123(49.0)$ & $1,442(49.3)$ & & $123(49.0)$ & $120(47.8)$ & \\
\hline Chemotherapy (\%) & & & 0.315 & & & 0.531 \\
\hline No/unknown & $241(96.0)$ & 2,759 (94.3) & & $241(96.0)$ & 237 (94.4) & \\
\hline Yes & $10(4.0)$ & $168(5.7)$ & & $10(4.0)$ & $14(5.6)$ & \\
\hline
\end{tabular}

PSM, propensity score matching; PN, partial nephrectomy; RN, radical nephrectomy; IQR, interquartile range; CCRCC, clear cell renal cell carcinoma; CHRCC, chromophobe renal cell carcinoma; PRCC, papillary renal cell carcinoma. 


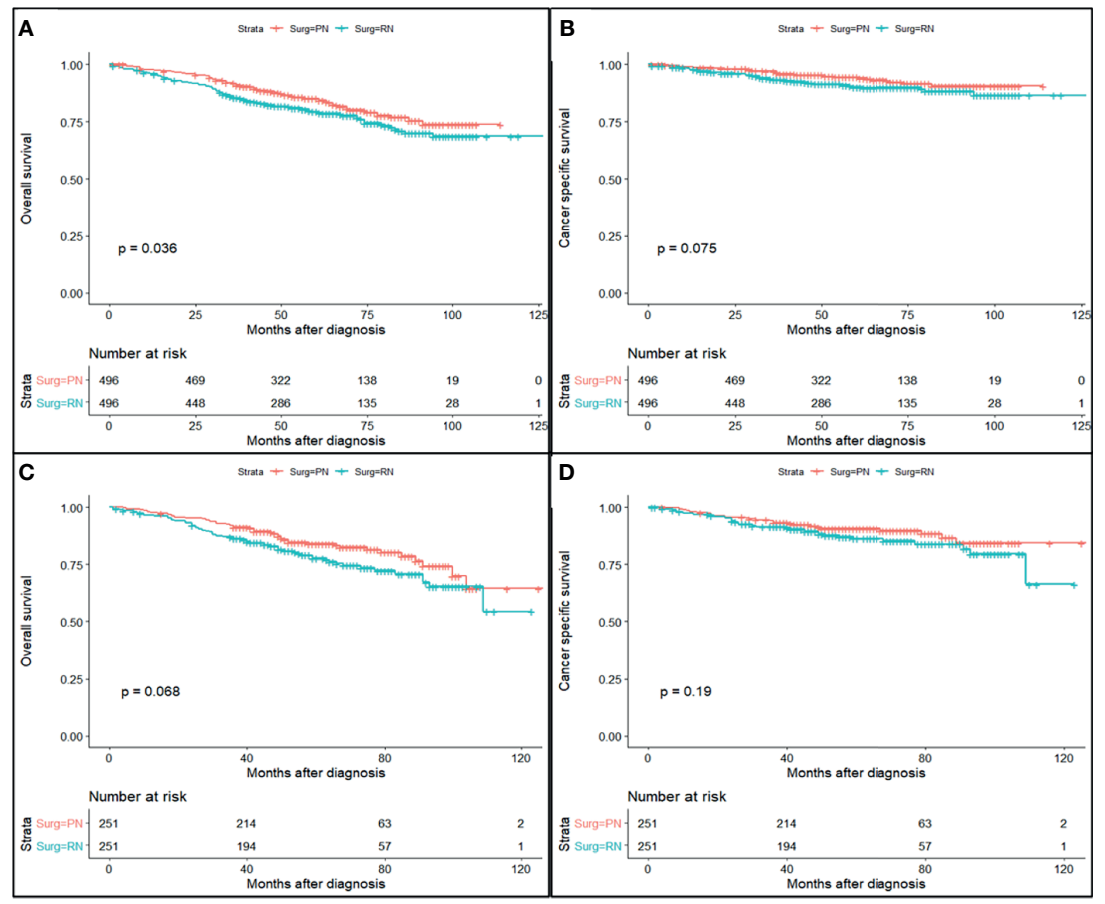

FIGURE 2 | Survival curve of partial nephrectomy (PN) and radical nephrectomy (RN) group in fat invasion cohort and venous invasion cohort after propensity score matching (PSM). (A) Overall survival (OS) curve of fat invasion cohort. (B) Cause-specific survival (CSS) curve of fat invasion cohort. (C) OS curve of venous invasion cohort. (D) CSS curve of venous invasion cohort.

age (HR 1.05, $p<0.001)$, greater tumor diameter (HR 1.02, $p=$ 0.007 ), and underwent systemic therapy ( $\mathrm{HR} 3.75, p=0.002)$; and pathological grade was also an independent prognostic factor $(p<0.001)$ (Table 5). Higher age was risk factor of both OS (HR 1.05, $p<0.001)$ and CSS (HR 1.04, $p=0.001)$ in the venous invasion cohort (Tables 6, 7). The surgical method had no significant effect on the OS $(p=0.069)$ and CSS $(p=0.190)$ of the venous invasion cohort and had no significant effect on the CSS $(p=0.078)$ of the fat invasion cohort (Table 8).

The median age of the TJH cohort was 53 (26-73) years; the $\mathrm{TJH}$ cohort included $57(87.7 \%)$ fat invasion and $8(12.3 \%)$ venous invasion patients, and 25 (38.5\%) PN and 40 (61.5\%) RN patients (Table 9). Postoperative estimated glomerular filtration rate (eGFR) was greater in patients receiving $\mathrm{PN}(p=0.027)$. The median follow-up was $30(1-105)$ months, 5 patients died at the last follow-up (all of them died of RCC), and 9 cases underwent cancer relapsed. The 1-, 3-, and 5-year OS rates (CSS same as OS) of the TJH cohort were $93.6 \%, 91.8 \%$, and $91.8 \%$, respectively; the 1-, 3-, and 5-year RFS rates were $90.2 \%, 85.7 \%$, and $82.2 \%$, respectively. KMA showed no significant difference in RFS between the RN and PN groups $(p=0.170)$ (Figure 4).

\section{DISCUSSION}

With the progression of the technology and the in-depth understanding of RCC, the indications for PN are gradually expanding, from initial $\mathrm{T} 1 \mathrm{a}$ to $\mathrm{T} 1 \mathrm{~b}$ and then to the current selected T2 $(5,16)$. It is reasonable to believe that PN will be provided to some appropriate $\mathrm{T} 3 \mathrm{a}$ patients in some technologically advanced institutions (12).

Even though urologists do not deliberately provide PN for T3a patients, they will encounter cases where cT1 and cT2 patients undergo PN upstage to pT3a. The postoperative upstaging occurs in approximately $11.35 \%$ of cT1 and cT2 patients; studies have shown that $\mathrm{RN}$ does not improve the RFS of upstaged patients as compared with PN (9). The study of Russell et al. included 1,955 cases with cT1 RCC who underwent PN, 95 of which upstaged to pT3a, and the PFS and CSS of pT3a patients were significantly worse than those of $\mathrm{pT} 1$ patients (both $p<0.01$ ) (17). The study of Groin et al. included 855 RCC patients who received $\mathrm{PN}, 41$ (4.8\%) of them upstaged to pT3a, and the recurrence rate of pT3a patients was significantly higher than that of pT1-2 patients at 2 years (99.2\% vs. 91.8\%) (18); these studies supported the current $\mathrm{T}$ staging but did not compare the prognosis of T3a patients with PN or RN. Shvero et al. compared 48 pT3a RCC patients who received PN with 86 patients who received $\mathrm{RN}$ and found that the surgical method was not significantly related to local recurrence, distant metastasis, CSS, or OS (10). Research by Andrade et al. and Deng et al. also drew similar conclusions $(10,19)$, but the study of Shah et al. showed that pT3a patients receiving PN are associated with shorter RFS $(p=0.001)$ (14). In our study's SEER cohort and the TJH cohort, the CSS or RFS of pT3a RCC 


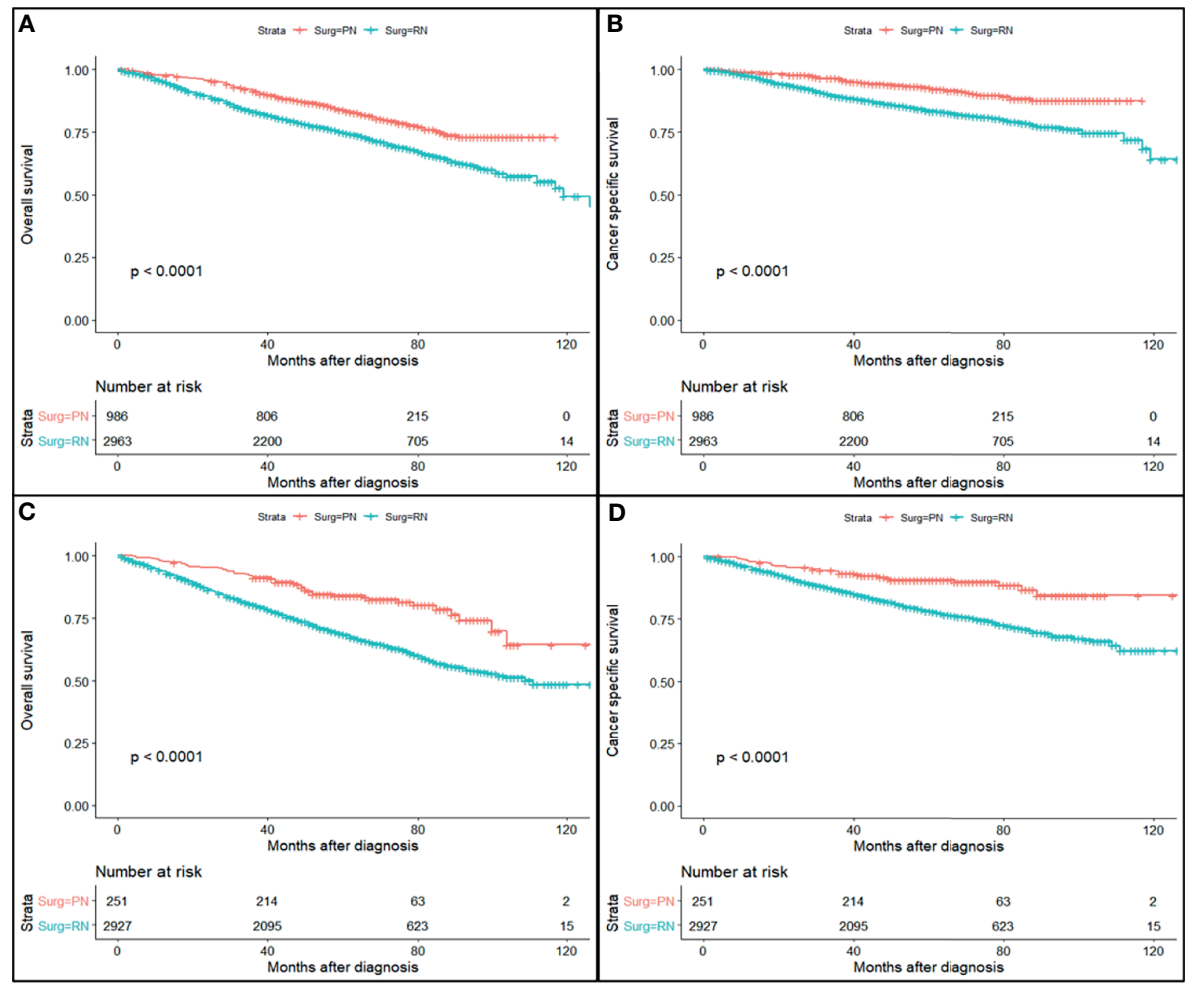

FIGURE 3 | Survival curve of partial nephrectomy (PN) and radical nephrectomy (RN) group in fat invasion cohort and venous invasion cohort before propensity score matching (PSM). (A) Overall survival (OS) curve of fat invasion cohort. (B) Cause-specific survival (CSS) curve of fat invasion cohort. (C) OS curve of venous invasion cohort. (D) CSS curve of venous invasion cohort.

TABLE 4 | Univariate and multivariate analyses of OS in the fat invasion cohort.

\begin{tabular}{|c|c|c|c|c|c|c|}
\hline Characteristics & \multicolumn{3}{|c|}{ Univariable analysis } & \multicolumn{3}{|c|}{ Multivariable analysis } \\
\hline No/unknown & 1 (Reference) & & & 1 (Reference) & & \\
\hline Yes & 2.33 & $1.09-4.95$ & 0.028 & 2.09 & $0.97-4.50$ & 0.06 \\
\hline Grade & & & 0.001 & & & 0.016 \\
\hline । & 1 (Reference) & & & 1 (Reference) & & \\
\hline IV & 4.22 & $1.72-10.33$ & 0.002 & 3.49 & $1.41-8.63$ & 0.007 \\
\hline Unknown & 1.74 & $0.72-4.24$ & 0.22 & 1.58 & $0.65-3.85$ & 0.315 \\
\hline Size & 1.01 & $1.00-1.02$ & $<0.001$ & 1.01 & $1.00-1.01$ & 0.007 \\
\hline \multicolumn{7}{|l|}{ Surgery } \\
\hline PN & 1 (Reference) & & & 1 (Reference) & & \\
\hline $\mathrm{RN}$ & 1.35 & $1.02-1.79$ & 0.037 & 1.35 & $1.01-1.79$ & 0.04 \\
\hline
\end{tabular}

OS, overall survival; PN, partial nephrectomy; RN, radical nephrectomy.

patients who received $\mathrm{PN}$ was not worse than that of patients who received $\mathrm{RN}$.

In addition to upstaged pT3a patients, some institutions have also tried to actively perform PN for cT3a patients. The study by Yim et al. included 159 cT3a RCC patients who received robot-assisted PN from multiple centers, of which $64.3 \%$ of the cases achieved a trifecta (negative surgical margins, warm ischemia time (WIT) $\leq 25 \mathrm{~min}$, and no perioperative complications), and $37.6 \%$ of patients achieved the optimal outcome (trifecta and $\geq 90 \%$ preservation of the eGFR and no stage upgrading of chronic kidney disease). The 5-year RFS, CSS, and OS were $82.1 \%, 93.3 \%$, and $91.3 \%$, respectively. The downside is that there is no $\mathrm{RN}$ patient as a control (12). 
TABLE 5 | Univariate and multivariate analyses of CSS in the fat invasion cohort.

\begin{tabular}{|c|c|c|c|c|c|c|}
\hline \multirow[t]{2}{*}{ Characteristics } & \multicolumn{3}{|c|}{ Univariable analysis } & \multicolumn{3}{|c|}{ Multivariable analysis } \\
\hline & Hazard ratio & Cl95. & $p$-Value & Hazard ratio & Cl95. & $p$-Value \\
\hline \multicolumn{7}{|l|}{ Chemotherapy } \\
\hline No/unknown & 1 (Reference) & & & 1 (Reference) & & \\
\hline Yes & 5.21 & $2.26-11.98$ & $<0.001$ & 3.75 & $1.6-8.78$ & 0.002 \\
\hline Grade & & & $<0.001$ & & & $<0.001$ \\
\hline I & 1 (Reference) & & & 1 (Reference) & & \\
\hline$\|$ & 1.42 & $0.33-6.08$ & 0.635 & 1.14 & $0.27-4.91$ & 0.856 \\
\hline III & 3.47 & $0.83-14.45$ & 0.088 & 2.1 & $0.5-8.87$ & 0.315 \\
\hline IV & 8.6 & $1.98-37.27$ & 0.004 & 5.43 & $1.24-23.72$ & 0.025 \\
\hline Unknown & 1.18 & $0.24-5.86$ & 0.837 & 0.94 & $0.19-4.66$ & 0.938 \\
\hline Size (per mm) & 1.02 & $1.01-1.03$ & $<0.001$ & 1.02 & $1.01-1.02$ & $<0.001$ \\
\hline
\end{tabular}

CSS, cause-specific survival.

TABLE 6 | Univariate and multivariate analyses of OS in the venous invasion cohort.

\begin{tabular}{|c|c|c|c|c|c|c|}
\hline \multirow[t]{2}{*}{ Characteristics } & \multicolumn{3}{|c|}{ Univariable analysis } & \multicolumn{3}{|c|}{ Multivariable analysis } \\
\hline & Hazard ratio & Cl95. & $p$-Value & Hazard ratio & Cl95. & $p$-Value \\
\hline Age (per year) & 1.05 & $1.03-1.07$ & $<0.001$ & / & l & / \\
\hline
\end{tabular}

OS, overall survival.

TABLE 7 | Univariate and multivariate analyses of CSS in the venous invasion cohort.

\begin{tabular}{|c|c|c|c|c|c|c|}
\hline \multirow[t]{2}{*}{ Characteristics } & \multicolumn{3}{|c|}{ Univariable analysis } & \multicolumn{3}{|c|}{ Multivariable analysis } \\
\hline & Hazard ratio & Cl95. & $p$-Value & Hazard ratio & Cl95. & $p$-Value \\
\hline Grade & & & 0.013 & & & 0.055 \\
\hline 1 & 1 (Reference) & & & 1 (Reference) & & \\
\hline$\|$ & 0.41 & $0.13-1.27$ & 0.124 & 0.48 & $0.15-1.48$ & 0.199 \\
\hline III & 0.85 & $0.3-2.43$ & 0.762 & 0.81 & $0.28-2.34$ & 0.699 \\
\hline IV & 1.73 & $0.52-5.76$ & 0.37 & 1.58 & $0.47-5.27$ & 0.46 \\
\hline Unknown & 0.38 & $0.1-1.54$ & 0.177 & 0.36 & $0.09-1.48$ & 0.158 \\
\hline Size (per mm) & 1.01 & $1-1.02$ & 0.018 & 1.01 & $1-1.02$ & 0.089 \\
\hline
\end{tabular}

CSS, cause-specific survival.

TABLE 8 | Association of nephrectomy type and survival (partial nephrectomy is reference).

\begin{tabular}{|c|c|c|c|c|c|c|c|c|c|c|c|c|}
\hline \multirow[t]{3}{*}{ Subgroup } & \multicolumn{6}{|c|}{ css } & \multicolumn{6}{|c|}{ os } \\
\hline & \multicolumn{3}{|c|}{ Univar } & \multicolumn{3}{|c|}{ Multivar } & \multicolumn{3}{|c|}{ Univar } & \multicolumn{3}{|c|}{ Multivar } \\
\hline & HR & $95 \% \mathrm{Cl}$ & $p$ & HR & $95 \% \mathrm{Cl}$ & $p$ & HR & $95 \% \mathrm{Cl}$ & $p$ & HR & $95 \% \mathrm{Cl}$ & $p$ \\
\hline Fat invasion cohort & 1.5 & $0.96-2.36$ & 0.078 & / & / & / & 1.35 & $1.02-1.79$ & 0.037 & 1.35 & $1.01-1.79$ & 0.04 \\
\hline VTT invasion cohort & 1.4 & $0.84-2.33$ & 0.19 & 1 & $/$ & 1 & 1.43 & $0.97-2.10$ & 0.069 & / & 1 & / \\
\hline
\end{tabular}

CSS, cause-specific survival; OS, overall survival; HR, hazard ratio; VTT, venous tumor thrombus.

Compared with RN, PN is positively correlated with the risk of having a positive surgical margin, and about $2 \%-8 \%$ of PN patients have a positive surgical margin (20). Morris et al. found that T3 RCC patients with positive margins after RN showed a trend of poorer OS, but it was not statistically significant (21). The study by Petros et al. showed that positive margins were associated with recurrence, metastasis, and worse OS in PN patients (22). However, Tabayoyong et al., Takagi et al., and
Kang et al. found that positive margins in patients with PN are not certainly translated into worse oncologic outcomes (23-25).

Some studies found that patients who received PN had better RFS and CSS than those who received RN and interpreted it as greater renal function preservation, which might be related to better oncologic outcomes $(26,27)$; the same trend was also observed in the TJH cohort in our study. Palacios et al. found that the unfavorable oncologic outcome was more related to the 
TABLE 9 | Characteristics of PN and RN groups in TJH cohorts.

\begin{tabular}{|c|c|c|c|}
\hline Characteristics & $\mathrm{PN}(p=25)$ & $\mathrm{RN}(p=40)$ & $p$ \\
\hline Sex & & & 0.290 \\
\hline Female & $6(24.0)$ & $15(37.5)$ & \\
\hline Male & $19(76.0)$ & $25(62.5)$ & \\
\hline Age (median [IQR]) & $53.00[46.00,59.00]$ & $52.50[45.75,62.00]$ & 0.766 \\
\hline BMI (median [IQR]) & $25.00[23.02,26.49]$ & $23.29[21.60,25.72]$ & 0.124 \\
\hline ASA score & & & 0.698 \\
\hline High & $2(8.0)$ & $5(12.5)$ & \\
\hline Low & $23(92.0)$ & $35(87.5)$ & \\
\hline Hemoglobin (median [IQR]) & $144.00[138.00,150.00]$ & $124.50[113.75,143.25]$ & 0.001 \\
\hline Albumin (median [IQR]) & $41.90[39.30,43.70]$ & $40.15[38.15,42.18]$ & 0.060 \\
\hline Serum_Cr (median [IQR]) & $80.00[66.00,93.00]$ & $80.50[66.50,96.00]$ & 0.777 \\
\hline Preoperative eGFR (median [IQR]) & $89.00[79.40,100.60]$ & $90.45[76.00,103.60]$ & 0.824 \\
\hline Postoperative eGFR (median [IQR]) & $78.90[57.30,86.70]$ & $60.2[51.10,68.35]$ & 0.027 \\
\hline Grade & & & 0.120 \\
\hline High & $4(16.0)$ & $15(37.5)$ & \\
\hline Low & $17(68.0)$ & $17(42.5)$ & \\
\hline Unknown & $4(16.0)$ & $8(20.0)$ & \\
\hline RENAL_score & & & 0.015 \\
\hline High & $3(12.0)$ & $6(15.0)$ & \\
\hline Low & $9(36.0)$ & $3(7.5)$ & \\
\hline Moderate & $13(52.0)$ & $31(77.5)$ & \\
\hline Laterality & & & 0.799 \\
\hline Left & $11(44.0)$ & $20(50.0)$ & \\
\hline Right & $14(56.0)$ & $20(50.0)$ & \\
\hline Approach & & & $<0.001$ \\
\hline Laparoscopic & $10(40.0)$ & $27(67.5)$ & \\
\hline Open & $0(0.0)$ & 7 (17.5) & \\
\hline Robotic & $15(60.0)$ & $6(15.0)$ & \\
\hline Histology & & & 0.579 \\
\hline CCRCC & 19 (76.0) & $27(67.5)$ & \\
\hline Non-CCRCC & $6(24.0)$ & $13(32.5)$ & \\
\hline Subgroup & & & 0.139 \\
\hline Fat invasion & $24(96.0)$ & $33(82.5)$ & \\
\hline Venous invasion & $1(4.0)$ & 7 (17.5) & \\
\hline Renal failure & & & 1.000 \\
\hline No & $24(96.0)$ & 38 (95.0) & \\
\hline Yes & $1(4.0)$ & $2(5.0)$ & \\
\hline
\end{tabular}

PN, partial nephrectomy; RN, radical nephrectomy; TJH, Tongji Hospital; IQR, interquartile range; BMI, body mass index; ASA, American Society of Anesthesiologists; eGFR, estimated glomerular filtration rate; CCRCC, clear cell renal cell carcinoma.

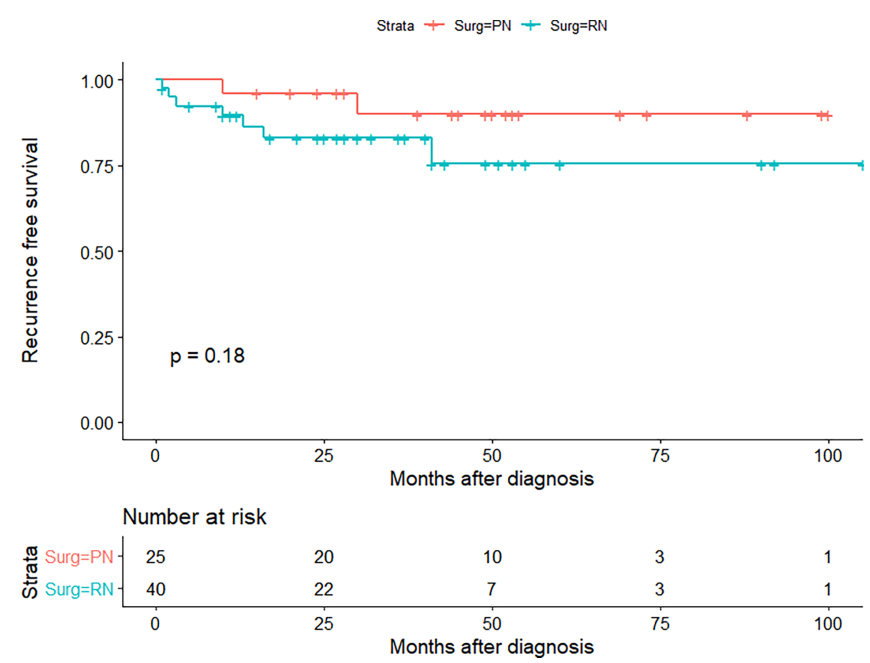

FIGURE 4 | Recurrence-free survival (RFS) curve of partial nephrectomy (PN) and radical nephrectomy (RN) group in Tongji Hospital (TJH) cohort. 
aggressive characteristics of the tumor itself, rather than the degree of renal function preservation (28). In our study, PN in the pre-PSM cohort was also significantly correlated with better CSS, but it was no longer significant after PSM. Therefore, we should cautiously interpret the trend that CSS and RFS of PN patients are better than those of RN patients in the study, which may be caused by retrospective study design and selection bias.

In view of the fact that some information such as hemoglobin, albumin, BMI, and comorbidities cannot be obtained from the SEER database, there were also some biases in this study, which may affect the accuracy of the conclusion.

\section{CONCLUSIONS}

This study proved that PN is safe and feasible in localized T3a RCC patients via a retrospective study with a large sample volume, and the oncologic outcomes of patients who underwent $\mathrm{PN}$ were comparable with those of patients who received $\mathrm{RN}$ but limited to pT3a patients, and higher-quality research is needed before exploring performing $\mathrm{PN}$ for cT3a RCC patients.

\section{DATA AVAILABILITY STATEMENT}

The raw data supporting the conclusions of this article will be made available by the authors, without undue reservation.

\section{REFERENCES}

1. Ferlay J, Steliarova-Foucher E, Lortet-Tieulent J, Rosso S, Coebergh JWW, Comber $\mathrm{H}$, et al. Cancer Incidence and Mortality Patterns in Europe: Estimates for 40 Countries in 2012. Eur J Cancer (Oxford England: 1990) (2013) 49(6):1374-403. doi: 10.1016/j.ejca.2012.12.027

2. Znaor A, Lortet-Tieulent J, Laversanne M, Jemal A, Bray F. International Variations and Trends in Renal Cell Carcinoma Incidence Andmortality. Eur Urol (2015) 67:519-30. doi: 10.1016/j.eururo.2014.10.002

3. Mari A, Tellini R, Antonelli A, Porpiglia F, Schiavina R, Amparore D, et al. A Nomogram for the Prediction of Intermediate Significant Renal Function Loss After Robot-Assisted Partial Nephrectomy for Localized Renal Tumors: A Prospective Multicenter Observational Study (RECORd2 Project). Eur Urol Focus (2021). doi: 10.1016/j.euf.2021.09.012

4. Carbonara U, Simone G, Capitanio U, Minervini A, Fiori C, Larcher A, et al. Robot-Assisted Partial Nephrectomy: 7-Year Outcomes. Minerva Urol Nephrol (2021) 73(4):540-3. doi: 10.23736/S2724-6051.20.04151-X

5. Ljungberg B, Albiges L, Abu-Ghanem Y, Bensalah K, Dabestani S, FernándezPello S, et al. European Association of Urology Guidelines on Renal Cell Carcinoma: The 2019 Update. Eur Urol (2019) 75(5):799-810. doi: 10.1016/ j.eururo.2019.02.011

6. Amparore D, Pecoraro A, Piramide F, Checcucci E, DE Cillis S, Volpi G, et al. Comparison Between Minimally-Invasive Partial and Radical Nephrectomy for the Treatment of Clinical T2 Renal Masses: Results of a 10-Year Study in a Tertiary Care Center. Minerva Urol Nephrol (2021) 73(4):509-17. doi: 10.23736/S2724-6051.21.04390-1

7. Stewart SB, Thompson RH, Psutka SP, Cheville JC, Lohse CM, Boorjian SA, et al. Evaluation of the National Comprehensive Cancer Network and American Urological Association Renal Cell Carcinoma Surveillance

\section{ETHICS STATEMENT}

The studies involving human participants were reviewed and approved by the Medical Ethical Committee of Tongji Hospital of Huazhong University of Science and Technology. Written informed consent for participation was not required for this study in accordance with the national legislation and the institutional requirements.

\section{AUTHOR CONTRIBUTIONS}

JT: data curation, writing-conceptualization, and original draft. XZ: formal analysis and supervision. JW: data curation and data analysis. JG: data curation and writing - review and editing. CK: software and supervision. WG: data analysis and supervision. $\mathrm{ZH}$ : project administration, resources, supervision, and funding acquisition. CY: writing-conceptualization, funding acquisition, and writing-review and editing. All authors contributed to the article and approved the submitted version.

\section{ACKNOWLEDGMENTS}

This work was supported by the National Natural Science Foundation of China (No. 81702989) and Sanming Project of Medicine in Shenzhen (No. SZSM202111003). The authors would like to thank Professor Kun Tang and Zhiqiang Chen (Department of Urology, Tongji Hospital, Tongji Medical College, Huazhong University of Science and Technology) for data curation.

Guidelines. J Clin Oncol: off J Am Soc Clin Oncol (2014) 32:4059-65. doi: 10.1200/JCO.2014.56.5416

8. Finelli A, Ismaila N, Bro B, Durack J, Eggener S, Evans A, et al. Management of Small Renal Masses: American Society of Clinical Oncology Clinical Practice Guideline. J Clin Oncol: Off J Am Soc Clin Oncol (2017) 35(6):66880. doi: 10.1200/JCO.2016.69.9645

9. Patel SH, Uzzo RG, Larcher A, Peyronnet B, Lane BR, Pruthi D, et al. Oncologic and Functional Outcomes of Radical and Partial Nephrectomy in Pt3a Pathologically Upstaged Renal Cell Carcinoma: A Multi-Institutional Analysis. Clin Genitourin Canc (2020) 18(6):e723-9. doi: 10.1016/j.clgc.2020.05.002

10. Shvero A, Nativ O, Abu-Ghanem Y, Zilberman D, Zaher B, Levitt M, et al. Oncologic Outcomes of Partial Nephrectomy for Stage T3a Renal Cell Cancer. Clin Genitourin Canc (2018) 16:e613-7. doi: 10.1016/j.clgc.2017.10.016

11. Andrade HS, Zargar H, Akca O, Kara O, Caputo PA, Ramirez D, et al. Is Robotic Partial Nephrectomy Safe for T3a Renal Cell Carcinoma? Experience of a High-Volume Center. J Endourol (2017) 31(2):153-7. doi: 10.1089/ end.2016.0622

12. Yim K, Aron M, Rha KH, Simone G, Minervini A, Challacombe B, et al. Outcomes of Robot-Assisted Partial Nephrectomy for Clinical T3a Renal Masses: A Multicenter Analysis. Eur Urol Focus (2020). doi: 10.1016/ j.euf.2020.10.011

13. Lee H, Lee M, Lee SE, Byun S, Kim HH, Kwak C, et al. Outcomes of Pathologic Stage T3a Renal Cell Carcinoma Up-Staged From Small Renal Tumor: Emphasis on Partial Nephrectomy. BMC Cancer (2018) 18(1):427. doi: 10.1186/s12885-018-4338-1

14. Shah PH, Moreira DM, Patel VR, Gaunay G, George AK, Alom M, et al. Partial Nephrectomy Is Associated With Higher Risk of Relapse Compared With Radical Nephrectomy for Clinical Stage T1 Renal Cell Carcinoma Pathologically Up Staged to T3a. J Urol (2017) 198:289-96. doi: 10.1016/j.juro.2017.03.012 
15. Cronin KA, Ries LAG, Edwards BK. The Surveillance, Epidemiology, and End Results (SEER) Program of the National Cancer Institute. Cancer Am Cancer Soc (2014) 120 Suppl 23:3755-7. doi: 10.1002/cncr.29049

16. Ljungberg B, Bensalah K, Canfield S, Dabestani S, Hofmann F, Hora M, et al. EAU Guidelines on Renal Cell Carcinoma: 2014 Update. Eur Urol (2015) 67 (5):913-24. doi: 10.1016/j.eururo.2015.01.005

17. Russell CM, Lebastchi AH, Chipollini J, Niemann A, Mehra R, Morgan TM, et al. Multi-Institutional Survival Analysis of Incidental Pathologic T3a Upstaging in Clinical T1 Renal Cell Carcinoma Following Partial Nephrectomy. Urology (2018) 117:95-100. doi: 10.1016/j.urology.2018.04.002

18. Gorin MA, Ball MW, Pierorazio PM, Tanagho YS, Bhayani SB, Kaouk JH, et al. Outcomes and Predictors of Clinical T1 to Pathological T3a Tumor UpStaging After Robotic Partial Nephrectomy: A Multi-Institutional Analysis. J Urol (2013) 190(5):1907-11. doi: 10.1016/j.juro.2013.06.014

19. Deng H, Fan Y, Yuan F, Wang L, Hong Z, Zhan J, et al. Partial Nephrectomy Provides Equivalent Oncologic Outcomes and Better Renal Function Preservation Than Radical Nephrectomy for Pathological T3a Renal Cell Carcinoma: A Meta-Analysis. Int Braz J Urol: Off J Braz Soc Urol (2021) 47 (1):46-60. doi: 10.1590/S1677-5538.IBJU.2020.0167

20. Choi JE, You JH, Kim DK, Rha KH, Lee SH. Comparison of Perioperative Outcomes Between Robotic and Laparoscopic Partial Nephrectomy: A Systematic Review and Meta-Analysis. Eur Urol (2015) 67(5):891-901. doi: 10.1016/j.eururo.2014.12.028

21. Morris LK, Altahan A, Gandhi J, Mays J, Giri U, Fleming M, et al. Impact of Margin Status on Survival After Radical Nephrectomy for Renal Cell Carcinoma. J Surg Oncol (2021) 123(2):687-92. doi: 10.1002/jso.26321

22. Petros FG, Metcalfe MJ, Yu K, Keskin SK, Fellman BM, Chang CM, et al. Oncologic Outcomes of Patients With Positive Surgical Margin After Partial Nephrectomy: A 25-Year Single Institution Experience. World J Urol (2018) 36(7):1093-101. doi: 10.1007/s00345-018-2241-7

23. Tabayoyong W, Abouassaly R, Kiechle JE, Cherullo EE, Meropol NJ, Shah ND, et al. Variation in Surgical Margin Status by Surgical Approach Among Patients Undergoing Partial Nephrectomy for Small Renal Masses. J Urol (2015) 194:1548-53. doi: 10.1016/j.juro.2015.06.076

24. Takagi T, Yoshida K, Wada A, Kondo T, Fukuda H, Ishihara H, et al. Predictive Factors for Recurrence After Partial Nephrectomy for Clinical T1 Renal Cell Carcinoma: A Retrospective Study of 1227 Cases From a Single
Institution. Int J Clin Oncol (2020) 25(5):892-8. doi: 10.1007/s10147-020 01632-x

25. Kang HW, Lee SK, Kim WT, Yun SJ, Lee S, Kim W, et al. Surgical Margin Does Not Influence Recurrence Rate in Pt1 Clear Cell Renal Cell Carcinoma After Partial Nephrectomy: A Multicenter Study. J Surg Oncol (2016) 114 (1):70-4. doi: 10.1002/jso.24259

26. Antonelli A, Minervini A, Sandri M, Bertini R, Bertolo R, Carini M, et al. Below Safety Limits, Every Unit of Glomerular Filtration Rate Counts: Assessing the Relationship Between Renal Function and Cancer-Specific Mortality in Renal Cell Carcinoma. Eur Urol (2018) 74(5):661-7. doi: 10.1016/j.eururo.2018.07.029

27. Antonelli A, Palumbo C, Sandri M, Veccia A, Furlan M, Zamboni S, et al. Renal Function Impairment Below Safety Limits Correlates With Cancer-Specific Mortality in Localized Renal Cell Carcinoma: Results From a Single-Center Study. Clin Genitourin Canc (2020) 18:e360-7. doi: 10.1016/j.clgc.2019.12.005

28. Palacios DA, Zabor EC, Munoz-Lopez C, Roversi G, Mahmood F, Abramczyk E, et al. Does Reduced Renal Function Predispose to Cancer-Specific Mortality From Renal Cell Carcinoma? Eur Urol (2021) 79(6):774-80. doi: 10.1016/ j.eururo.2021.02.035

Conflict of Interest: The authors declare that the research was conducted in the absence of any commercial or financial relationships that could be construed as a potential conflict of interest.

Publisher's Note: All claims expressed in this article are solely those of the authors and do not necessarily represent those of their affiliated organizations, or those of the publisher, the editors and the reviewers. Any product that may be evaluated in this article, or claim that may be made by its manufacturer, is not guaranteed or endorsed by the publisher.

Copyright (c) 2022 Tian, Zeng, Wan, Gan, Ke, Guan, Hu and Yang. This is an openaccess article distributed under the terms of the Creative Commons Attribution License (CC BY). The use, distribution or reproduction in other forums is permitted, provided the original author(s) and the copyright owner(s) are credited and that the original publication in this journal is cited, in accordance with accepted academic practice. No use, distribution or reproduction is permitted which does not comply with these terms. 